\section{MS43-P3 An Add-on Device for Automated In Situ Screening}

Angela Criswell ${ }^{1}$, Colin Acheson ${ }^{1}$, Pierre Le Magueres ${ }^{1}$, Thom Hendrixson ${ }^{1}$, Cheng Yang ${ }^{2}$, Zhao Zijian ${ }^{3}$, Joseph D. Ferrara ${ }^{1}$

1. Rigaku Oxford Diffraction

2. Beijing Liangjia Deshi Technology Ltd. Co.

3. Rigaku Beijing Corp

email: angela.criswell@rigaku.com

In situ screening of crystallization trials continues to be a topic of importance. In situ screening allows one to determine if the crystal-like object in a trial is salt, protein or something else of interest. Furthermore, crystal quality can be assessed before cryoprotection is attempted, providing a means to better optimize this step. Finally, some crystals are well-behaved enough that complete or nearly-complete data sets can be collected in situ, eliminating the need for harvesting and cryoportection altogether.

In this presentation we will show results from a newly developed automated in situ screening system designed specifically to work with legacy Rigaku diffractometers, extending their utility. The system is easy to install and deinstall so one can go from collecting data on cryo-protected samples to in situ screening in minutes. The system uses the same software as the Rigaku Oxford Diffraction PXScanner, CrystalEyes, making the transition between dedicated and part time systems easy.

Keywords: in situ crystallography
MS43-P4 Enol-imine/keto-amine tautomerism of

(E)-4-[(2-hydroxybenzylidene)amino]phenyl benzenesulfonate

\author{
Muharrem Dinçer ${ }^{1}$, Namık Özdemir ${ }^{2}$, Reyhan $\mathrm{Kağ}^{3}{ }^{3}$, Osman \\ Dayan $^{3}$
}

1. Department of Physics, Faculty of Arts and Sciences, Ondokuz Mayıs University, 55139, Samsun, Turkey

2. Department of Secondary School Science and Mathematics Education, Faculty of Education, Ondokuz Mayıs University, 55139, Samsun, Turkey

3. Department of Chemistry, Faculty of Arts and Sciences, Çanakkale Onsekiz Mart University, 17020, Çanakkale, Turkey

email: mdincer@omu.edu.tr

The title Schiff base compound has been obtained from the reaction of 4-aminophenyl benzenesulfonate and salicylaldehyde, and characterized by spectroscopic and single-crystal X-ray diffraction techniques. Quantum chemical calculations employing density functional theory (DFT) method with the 6-311++G(d,p) basis set were performed to study the molecular, spectroscopic and enol-imine/keto-amine tautomerization mechanism of the compound. There exists a good correlation between experimental and theoretical data. Thetautomerization mechanism was investigated in the gas phase and in solution phase using the polarizable continuum model (PCM) approximation. The energetic and thermodynamic parameters of the enol-imine $\rightarrow$ keto-amine transfer process show that the single proton exchange is unfavored in all cases. However, the reverse reaction seems to be feasible with a very low barrier height and is supported by negative values in enthalpy and free energy changes for all cases.

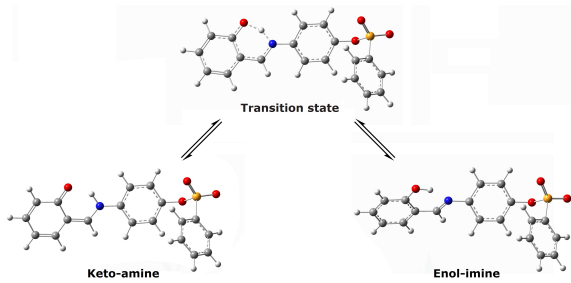

Figure 1. Tautomerizationof the title compound.

Keywords: Crystal structure, DFT, Enol-imine/keto-amine tautomerism 